\title{
Tailored nanoscale plasmon-enhanced vibrational electron spectroscopy
}

Luiz H. G. Tizei ${ }^{1}$, Vahagn Mkhitaryan ${ }^{2}$, Hugo Lourenço-Martins ${ }^{3}$, Leonardo Scarabelli ${ }^{4}$, Kenji Watanabe $^{5}$, Takashi Taniguchi ${ }^{5}$, Marcel Tencé ${ }^{6}$, Jean-Denis Blazit ${ }^{7}$, Xiaoyan Li $^{8}$, Steffi Y. Woo ${ }^{9}$, Alexandre Gloter ${ }^{3}$, Alberto Zobelli ${ }^{10}$, Franz Schmidt ${ }^{11}$, F. Javier Garcia de Abajo ${ }^{12}$, Luis M. LizMarzán ${ }^{4}$, Odile Stéphan ${ }^{7}$ and Mathieu Kociak ${ }^{13}$

${ }^{1}$ Université Paris-Saclay, CNRS, Laboratoire de Physique des Solides, 91405, Orsay, France, France, ${ }^{2}$ ICFO, United States, ${ }^{3} \mathrm{CNRS}$, United States, ${ }^{4} \mathrm{CIC}$ biomaGUNE, United States, ${ }^{5}$ National Institute for Material Science, Tsukuba, Japan, ${ }^{6}$ Université Paris-Saclay, CNRS, Laboratoire de Physique des Solides, 91405, Orsay, France, ORSAY, France, ${ }^{7}$ Université Paris-Saclay, CNRS, Laboratoire de Physique des Solides, 91405, Orsay, France, United States, ${ }^{8}$ Université Paris-Saclay, CNRS, Laboratoire de Physique des Solides, 91405, Orsay, France, Ile-de-France, United States, ${ }^{9}$ Université Paris-Saclay, CNRS, Laboratoire de Physique des Solides, United States, ${ }^{10}$ Laboratoire de Physique des Solides/CNRS, Université Paris-Saclay, France, ${ }^{11}$ University of Graz, United States, ${ }^{12}$ ICFO-Institut de Ciencies Fotoniques, Castelldefels, Catalonia, Spain, ${ }^{13} \mathrm{CNRS}$, Orsay, Ile-de-France, United States

Atomic vibrations and phonons are an excellent source of information on nanomaterials that we can access through a variety of methods including Raman scattering, infrared spectroscopy, and electron energy-loss spectroscopy (EELS). In the presence of a plasmon local field, vibrations are strongly modified and, in particular, their dipolar strengths are highly enhanced, thus rendering Raman scattering and infrared spectroscopy extremely sensitive techniques.

In this contribution [1], we experimentally demonstrate that the interaction between a relativistic electron and vibrational modes in nanostructures is fundamentally modified in the presence of plasmons. This could be observed due to the current developments in electron optics, making possible the generation of sub-nanometer wide sub-10 meV electron beams with $60 \mathrm{keV}$ primary energy. We finely tune the energy of surface plasmons in metallic micrometer-long Ag nanowires in the vicinity of hexagonal boron nitride flakes, making it possible to monitor and disentangle both strong phonon-plasmon coupling and plasmondriven phonon enhacement at the nanometer scale. Because of the near-field character of the electron beam-phonon interaction, optically inactive phonon modes are also observed. Besides increasing our understanding of phonon physics, our results hold great potential for investigating sensing mechanisms and chemistry in complex nanomaterials down to the molecular level.

The strong coupling limit is reached between surface plasmons and phonon modes (Figure 1a) as the largest coupling constant, $g$, reached was $52 \mathrm{meV}$ (Figure 1b) and the full width at half maximum of the two coupled excitations were 23 and $19 \mathrm{meV}$. The signature of strong coupling is observed along the whole nanowires, including for nanowire regions far away from any hBN (point to the coherence of the coupled modes along the nanowire). The spatial dependence at nanometer scale of these modes will be described.

More interestingly, the presence of plasmons modify the way fast electrons couple to bulk modes of $\mathrm{hBN}$, leading to enhancement of their signal and the observation of usually "dark modes". As an example, the ZO-phonon mode of $\mathrm{hBN}$, usually not observed for electron beams perpendicularly incident on an 
hBN flake, can be observed when close to metallic nanowires (Figure 1c). We will describe how this enhanced signature changes as a function of the distance of the electron probe to the nanowire surface, varying in the tens-of-nanometers scale. Experiments have been performed with the ChromaTEM microscope (TEMPOS project, ANR-10-EQPX-50), a modified Nion HERMES200 operated at $60 \mathrm{kV}$. The electron beam was set to have an energy spread in the 6-10 meV range and a nanometer size. Experiments are under way to achieve similar enhancement in molecular systems.
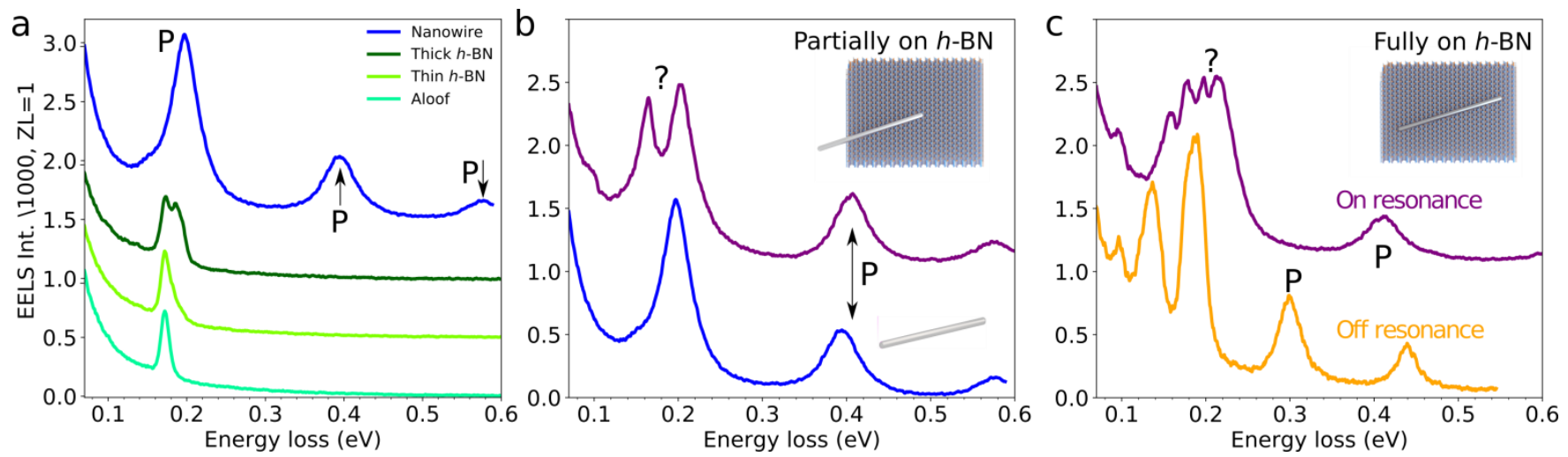

Figure 1. a) EELS of isolated Ag nanowires (blue) and hBN of different thicknesses (green). b) EELS spectra of two nanowires of similar lenghts, one isolated (blue) and one in strong coupling condition (purple). c) EELS spectra of a nanowire in and out of resonance close to hBN, showing enhancement of phonon modes. This figure has been reproduced partially from [1].

References

[1] Tizei, L. H. G., et al, 2020, Nano Lett. 20, 2973 (2020). 